Journal of Molecular Structure, 87 (1982) 221-227

THEOCHEM

Elsevier Scientific Publishing Company, Amsterdam - Printed in The Netherlands

\title{
AN AB INITIO STUDY OF THE STRUCTURE AND TORSIONAL MODES OF THE $\mathrm{H}_{2} \mathrm{SO}_{4}$ MOLECULE
}

\author{
LAWRENCE L. LOHR, JR.
}

Department of Chemistry, University of Michigan, Ann Arbor, Michigan 48109 (U.S.A.)

(Received 27 May 1981)

\begin{abstract}
$A b$ initio calculations at the STO-3G and 4-31G levels have been carried out for the $\mathrm{H}_{2} \mathrm{SO}_{4}$ molecule as a function of the pair of twist angles of the $\mathrm{H}-\mathrm{O}$ bonds about the respective $\mathrm{O}-\mathrm{S}$ bonds. Values for the remaining bond angles and lengths were taken from the recent microwave structural determination by Kuczkowski et al. The results indicate a minimum energy for a structure with a (sc, sc) conformation and $C_{2}$ symmetry, where sc denotes synclinal, or gauche. This structure corresponds to that observed. At a higher energy of $11.5 \mathrm{~kJ} \mathrm{~mol}^{-1}(4-31 \mathrm{G})$ there is a structure with a $(+\mathrm{sc},-\mathrm{sc})$ conformation and $C_{\mathrm{s}}$ symmetry. The torsional modes corresponding to the $a$ and $b$ irreducible representations of the $C_{2}$ point group are estimated to have frequencies of 280 and $265 \mathrm{~cm}^{-1}$, respectively.
\end{abstract}

\section{INTRODUCTION}

The detailed structure of the gaseous $\mathrm{H}_{2} \mathrm{SO}_{4}$ molecule has recently been determined [1] from the microwave spectra of four isotopic species. The structure is characterized by $C_{2}$ symmetry, with the conformation of the OH groups being $(+s c,+s c)$, where "sc" denotes synclinal (gauche). This observed conformation differs from the $C_{2 \mathrm{v}}$ (ap, ap) conformation, where "ap" denotes antiperiplanar (trans), predicted [2] from CNDO/2 calculations. We report here the results of an ab initio study of the molecule at the singledeterminant STO-3G and 4-31G levels using the program Gaussian-70 [3]. The principal result is the location of a minimum energy structure with a $(+\mathrm{sc},+\mathrm{sc})$ conformation and $C_{2}$ symmetry, in agreement with experiment.

The observed structure [1] is characterized by the distances (in $\AA$ ) $\mathrm{S}-\mathrm{O}_{1}$ $=1.574 \pm 0.01, \mathrm{~S}-\mathrm{O}_{2}=1.422 \pm 0.01, \mathrm{O}_{1}-\mathrm{H}_{1}=0.97 \pm 0.01$, by the bond angles (in degrees) $\mathrm{O}_{1}-\mathrm{S}-\mathrm{O}_{1}^{\prime}=101.3 \pm 1, \mathrm{O}_{2}-\mathrm{S}-\mathrm{O}_{2}^{\prime}=123.3 \pm 1, \mathrm{H}_{1}-$ $\mathrm{O}_{1}-\mathrm{S}=108.5 \pm 1.5$, and by an $\mathrm{H}_{1}-\mathrm{O}_{1}-\mathrm{S}-\mathrm{O}_{1}^{\prime}$ dihedral angle of $-90.9 \pm 1^{\circ}$. This dihedral angle corresponds to a value of $89.1^{\circ}$ for both $\phi$ and $\phi^{\prime}$ in Fig. 1. In addition, the angle $\mathrm{P}_{1} \mathrm{P}_{2}$ between the $\mathrm{O}_{1} \mathrm{SO}_{1}^{\prime}$ and $\mathrm{O}_{2} \mathrm{SO}_{2}^{\prime}$ planes is $88.4 \pm 0.1^{\circ}$, with this small deviation from $90^{\circ}$ bringing the atoms $\mathrm{O}_{2}$ and $\mathrm{O}_{2}^{\prime}$ closer to $\mathrm{O}_{1}^{\prime}$ and $\mathrm{O}_{1}$, respectively.

In our ab initio studies we have assumed the values of the three bond lengths and three bond angles given above, and have explored the variation 


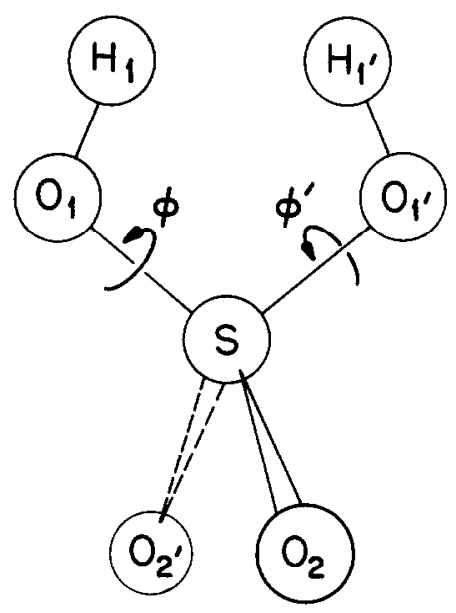

Fig. 1. Atomic designations for the $\mathrm{H}_{2} \mathrm{SO}_{4}$ molecule. The variables considered in this study are the twist angles $\phi$ and $\phi^{\prime}$, shown here for the energy maximum at $\phi=\phi^{\prime}=180^{\circ}$, corresponding to the $(p, p)$ structure, where " $p$ " denotes periplanar, or cis.

of the total energy with respect to the pair of twist angles $\phi$ and $\phi^{\prime}$. For most of our studies the angle $P_{1} P_{2}$ has been set equal to $90^{\circ}$, since only a small difference in energy has been found between the observed structure with the angle $P_{1} P_{2}=88.4^{\circ}$ and one identical except with the angle $P_{1} P_{2}=$ $90^{\circ}$ (0.2 $\mathrm{kJ} \mathrm{mol}^{-1}$ favoring the latter).

\section{POTENTIAL ENERGY SURFACE}

Using a grid of points corresponding to $30^{\circ}$ increments of $\phi$ and $\phi^{\prime}$, we first generated the STO-3G potential energy surface shown in Fig. 2. The mutually perpendicular dashed lines represent mirror planes of symmetry for the surface, so that the grid of 169 points is constructed from 43 calculated points. The global minimum on this surface has $C_{2}$ symmetry and a (+sc, $+\mathrm{sc}$ ) conformation with $\phi=\phi^{\prime}=81.4^{\circ}$, somewhat smaller than the observed value of $\phi=89.1^{\circ}$. There is also a local minimum with $C_{s}$ symmetry and a $\left(+\mathrm{sc},-\mathrm{sc}\right.$ ) conformation with $\phi=-\phi^{\prime}=76.0^{\circ}$ and an energy of $5.7 \mathrm{~kJ}$ $\mathrm{mol}^{-1}$ relative to the $C_{2}$ minimum. In locating these we made use of four additional calculations with $5^{\circ}$ increments in the twist angles with the constraints $\phi=\phi^{\prime}$ for $C_{2}$ symmetry and $\phi=-\phi^{\prime}$ for $C_{\mathrm{s}}$ symmetry. The saddle point between these minima has a ( $\pm \mathrm{sc}$, ap) conformation with approximate twist angles $\phi=90^{\circ}, \phi^{\prime}=0^{\circ}$ and an energy of $14.0 \mathrm{~kJ} \mathrm{~mol}^{-1}$. The $( \pm \mathrm{sc}, \mathrm{p})$ saddle point, where " $p$ " denotes periplanar, or cis, near $\phi=60^{\circ}, \phi^{\prime}=180^{\circ}$ is at a higher energy, namely $17.4 \mathrm{~kJ} \mathrm{~mol}^{-1}$. Both the (ap, ap) and $(\mathrm{p}, \mathrm{p}) C_{2 \mathrm{v}}$ structures are local maxima, with energies of 30.1 and $70.6 \mathrm{~kJ} \mathrm{~mol}^{-1}$, respectively. These results are summarized in Table 1 . In contrast to the CNDO/2 results [2], the (ap, ap) structure is clearly excluded as the equilibrium geometry. We also note that whereas least motion racemization lead through the 


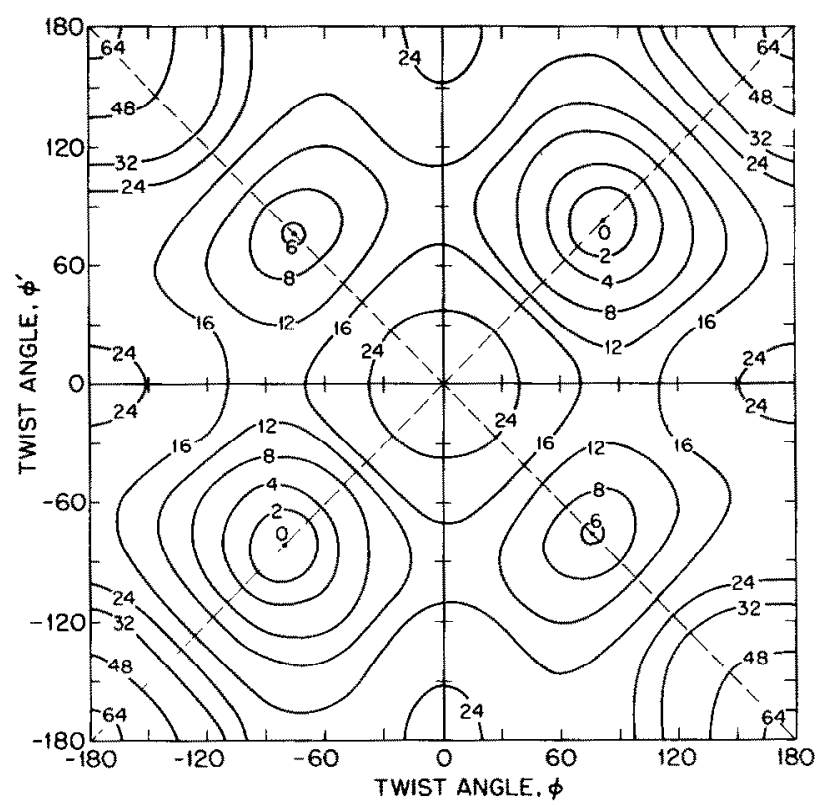

Fig. 2. Total energy vs. $\phi$ and $\phi^{\prime}$ for $\mathrm{H}_{2} \mathrm{SO}_{4}$ at the STO-3G level. The numbers on the contours denote values of the energy in $\mathrm{kJ} \mathrm{mol}^{-1}$ relative to the energy of the $(+\mathrm{sc},+\mathrm{sc})$ conformation with $\phi=\phi^{\prime}=81.3^{\circ}$. The lowest energy computed point is at $\phi=\phi^{\prime}=81.4^{\circ}$, where the STO-3G total energy is -689.23602 au.

high energy $C_{2 \mathrm{v}}$ maxima, the lowest energy racemization pathway corresponds to "circular" motion (Fig. 2) through the $C_{s}$ local minima and two $( \pm \mathrm{sc}, \mathrm{ap})$ saddle points previously described.

In order to support the above conclusions, calculations at eleven selected points in $\phi, \phi^{\prime}$ space were done using the larger 4-31G basis set, with the key results summarized in Table 2 . The structure with $C_{2}$ symmetry has a minimum energy for $\phi=\phi^{\prime}=85.1^{\circ}$, somewhat closer to the observed value of $89.1^{\circ}$ than is the STO-3G value of $81.3^{\circ}$. The structure with $C_{s}$ symmetry

\section{TABLE 1}

STO-3G Energies for $\mathrm{H}_{2} \mathrm{SO}_{4}$

\begin{tabular}{llrrr}
\hline Symmetry & Extremum & $\phi\left(^{\circ}\right)$ & $\phi^{\prime}\left(^{\circ}\right)$ & $\begin{array}{l}\Delta E \\
\left(\mathrm{~kJ} \mathrm{~mol}^{-1}\right)^{\mathbf{a}}\end{array}$ \\
\hline$C_{2}$ & Min & 81.3 & 81.3 & 0.0 \\
$C_{\mathrm{s}}$ & Min & 75.9 & -75.9 & 5.7 \\
$C_{5}$ & Saddle & 0.0 & 87.5 & 14.0 \\
$C_{1}$ & Saddle & 180.0 & 60.0 & 17.4 \\
$C_{2 \mathrm{v}}$ & Max & 0.0 & 0.0 & 30.1 \\
$C_{2 \mathrm{v}}$ & Max & 180.0 & 180.0 & 70.6 \\
$C_{\mathrm{s}}$ & Max & 0.0 & 180.0 & 26.2 \\
\hline
\end{tabular}

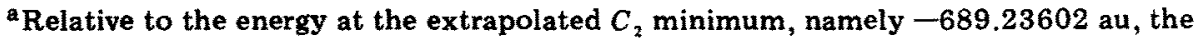
same value as that computed for $\phi=\phi^{\prime}=81.4^{\circ}$. 
TABLE 2

4-31G Energies for $\mathrm{H}_{2} \mathrm{SO}$,

\begin{tabular}{lcrc}
\hline Symmetry & $\phi\left(^{\circ}\right)$ & $\phi^{\prime}\left(^{\circ}\right)$ & \multicolumn{1}{|c}{$\begin{array}{l}\Delta E \\
\left(\mathbf{k J ~ m o l} \mathbf{~ m o l}^{-1}\right.\end{array}$} \\
\hline$C_{2}$ & $85.1^{\mathrm{b}}$ & 85.1 & 0.0 \\
$C_{\mathrm{s}}$ & $70.0^{\mathrm{b}}$ & -70.0 & 11.5 \\
$C_{1}$ & 0.0 & 87.5 & 10.1 \\
$C_{2 \mathrm{v}}$ & 0.0 & 0.0 & 23.8 \\
$C_{2 \mathrm{v}}$ & 180.0 & 180.0 & 101.0 \\
\hline
\end{tabular}

${ }^{a}$ Relative to the computed energy at the extrapolated $C_{2}$ minimum, namely -696.90154 au for $\phi=\phi^{\prime}=85.1^{\circ}$.

bValues found by minimization of energy with respect to $\phi$.

is found to have a minimum energy for $\phi=-\phi^{\prime}=70.0^{\circ}$, somewhat smaller than the STO-3G value of $75.9^{\circ}$. The energy at the $C_{\mathrm{s}}$ minimum is $11.5 \mathrm{~kJ}$ $\mathrm{mol}^{-1}$ higher than that at the $C_{2}$ minimum, an energy difference twice as great as that found in the STO-3G calculations, but nonetheless below that of either the structures with $C_{2 v}$ symmetry (Table 2). However, we have not directly demonstrated that the two $C_{2 \mathrm{v}}$ structures are local maxima in $\phi, \phi^{\prime}$ space at the 4-31G level. Similarly, while the $C_{\mathrm{s}}$ structure is clearly a local minimum at the STO-3G level (Fig. 2), the $4-31 \mathrm{G}$ energy of $11.5 \mathrm{~kJ} \mathrm{~mol}^{-1}$ is slightly above the $10.1 \mathrm{~kJ} \mathrm{~mol}^{-1}$ value (Table 2) for a $C_{1}$ structure with $\phi=0.0^{\circ}, \phi^{\prime}=87.5^{\circ}$, which is the approximate location of a saddle point at the STO-3G level. Thus the $4-31 \mathrm{G}$ results suggest that while the $C_{\mathrm{s}}$ structure has a minimum $\left(\phi=-\phi^{\prime}=70.0^{\circ}\right)$ with respect to its totally symmetric $\left(a^{\prime}\right)$ displacement mode in $\phi, \phi^{\prime}$ space, it is either a shallow minimum or possibly a maximum with respect to its antisymmetric $\left(a^{\prime \prime}\right)$ displacement mode.

\section{TORSIONAL VIBRATIONS}

There are two torsional modes of vibration for the $\mathrm{H}_{2} \mathrm{SO}_{4}$ molecule with $C_{2}$ symmetry, these corresponding to $a$ and $b$ irreducible representations. From the 4-31G level energies computed at the cxtrapolated minimum $\left(\phi=\phi^{\prime}=85.1^{\circ}\right)$ and for structures slightly displaced along each symmetry coordinate, we obtain molecular force constants of $6.90 \times 10^{-3}$ and $6.25 \times$ $10^{-3} \mathrm{~kJ} \mathrm{~mol}^{-1} \mathrm{deg}^{-2}$ for the $a$ and $b$ modes, respectively (these are second derivatives of the total electronic energy, including nuclear-nuclear repulsion). Using a projected $\mathrm{O}-\mathrm{H}$ of $0.92 \AA$, these values become $4.45 \times 10^{+3}$ and $4.02 \times 10^{+3} \mathrm{erg} \mathrm{cm}^{-2}$, respectively, which, when combined with a reduced mass taken as $1 \mathrm{amu}$, yield harmonic frequencies of 280 and $265 \mathrm{~cm}^{-1}$ for the $a$ and $b$ modes, respectively. These modes may be described as radial and tangential displacements with respect to the $C_{2}$ symmetry minima (Fig. 2). 
Since the surface in Fig. 2 is periodic in $2 \pi$ for both $\phi$ and $\phi^{\prime}$, we consider the closure of this surface to form a torus, which is the appropriate representation given this periodicity. The closure may be visualized by joining the top and bottom horizontal edges to form a cylinder, after which the rightand left-hand circular ends are joined, completing the torus. The torsional motions and racemization of $\mathrm{H}_{2} \mathrm{SO}_{4}$ may then be viewed as motions of a mass point on the surface of the torus with a position-dependent potential energy. A similar portrayal [4] has been given for the ring deformations of cycloheptane.

\section{DISCUSSION}

The results of this study resemble those found for other $\mathrm{AB}_{2}(\mathrm{OR})_{2}$ or $\mathrm{A}(\mathrm{OR})_{2}$ systems, such as $\mathrm{CH}_{2}(\mathrm{OH})_{2}[5,6], \mathrm{O}(\mathrm{OH})_{2}[5]$, and $\left[\mathrm{PO}_{2}\left(\mathrm{OCH}_{3}\right)_{2}^{-}\right]$

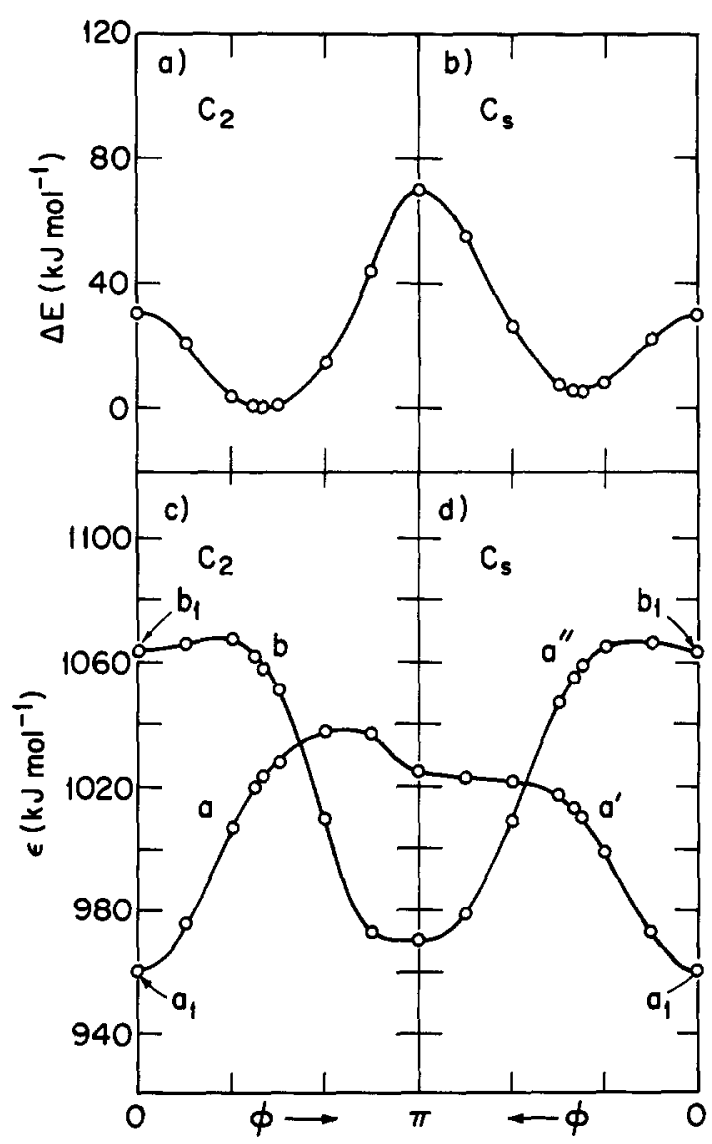

Fig. 3. Total energy vs. $\phi$ for the $C_{2}$-symmetry isomer (a) and the $C_{s^{-s y m m e t r y}}$ isomer (b) at the STO-3G level, together with energies of the $a$ and $b$ LUMO's for the $C_{\mathrm{s}}$-symmetry isomer (c) and energies of the $a^{\prime}$ and $a^{\prime \prime}$ LUMO's for the $C_{\mathrm{s}}$-symmetry isomer (d). All energies are in $\mathrm{kJ} \mathrm{mol}^{-1}$. 
TABLE 3

Energies of valence shell molecular orbitals for $\mathrm{H}_{2} \mathrm{SO}_{4}$

\begin{tabular}{llllll}
\hline Number & Symmetry $^{\mathrm{a}}$ & $-\epsilon(\mathrm{eV})^{\mathrm{b}}$ & Number & Symmetry $^{\mathrm{a}}$ & $-\epsilon(\mathrm{eV})^{\mathrm{b}}$ \\
\hline 1 & $a$ & 38.32 & 9 & $a$ & 16.44 \\
2 & $b$ & 34.45 & 10 & $b$ & 15.02 \\
3 & $a$ & 33.79 & 11 & $a$ & 14.95 \\
4 & $b$ & 33.55 & 12 & $b$ & 13.80 \\
5 & $a$ & 22.51 & 13 & $a$ & 12.79 \\
6 & $b$ & 20.10 & 14 & $b$ & 12.63 \\
7 & $a$ & 18.03 & 15 & $a$ & 12.41 \\
8 & $b$ & 17.62 & 16 & $b$ & $12.09^{c}$ \\
\hline
\end{tabular}

${ }^{2}$ Representation of the group $C_{2}$.

bValues from 4-31G computation at $C_{2}$ minimum with $\phi=\phi^{\prime}=85.1^{\circ}$.

cHOMO; the $a$ and $b$ virtual LUMO energies are +3.37 and $+4.15 \mathrm{eV}$, respectively.

[7], in that a structure of $C_{2}$ rather than $C_{2 \mathrm{v}}$ symmetry corresponds to the equilibrium geometry. The instability of the $C_{2 v}$ forms of $\mathrm{H}_{2} \mathrm{SO}_{4}$ is emphasized in Fig. 3(a), which shows the variation of the total energy $E$ (STO-3G level) with respect to $\phi$ for $C_{2}$ structures $\left(\phi=\phi^{\prime}\right)$. The symmetry $C_{2 v}$ obtains for $\phi=0$ (ap, ap) and for $\phi=\pi$ (p, p). Correspondingly in Fig. 3(c) are shown the energies $\epsilon$ of the lowest virtual (empty) orbitals of $a$ and $b$ symmetries, these orbitals both being largely $\mathrm{OH}$ antibonding in character. $\mathrm{A}$ decrease in $E$ is clearly associated with increases in $\epsilon$, reflecting the collective increased stabilization of occupied orbitals of the same symmetries. Similar results obtain for the variation of $E$ with respect to $\phi$ for $C_{s}$ structures ( $\phi=$ $\left.-\phi^{\prime}\right)$, Fig. 3(b), with the energies of the $a^{\prime}$ and $a^{\prime \prime}$ virtual orbitals being shown in Fig. 3(d). The $C_{2}, C_{s}$ and $C_{2 \mathrm{v}}$ (ap, ap) structures may all be thought of as being derived from eclipsed $\mathrm{SO}_{2}\left(\mathrm{CH}_{3}\right)_{2}$ with $\mathrm{OH}$ replacing $\mathrm{CH}_{3}$. The computed energy ordering of these structures then reflects the destabilizing effects of interactions between lone pairs on different oxygen atoms.

In Table 3 we present the energies of the valence shell molecular orbitals at the 4-31G level for the $C_{2}$ symmetry equilibrium geometry $\left(\phi=\phi^{\prime}=\right.$ $\left.85.1^{\circ}\right)$. The first four of these levels are predominantly $O(2 s)$ in character, while the $b$ HOMO (no. 16) and the $a$ level below it (no. 15) are predominantly $\mathrm{O}(2 p)$ lone pairs on the $\mathrm{O}$ atoms without attached $\mathrm{H}$ atoms.

Interestingly, the Mulliken charge distribution indicates little difference between the two types of $O$ atoms, the computed charges being -0.81 and -0.80 for the $\mathrm{O}$ 's with and without attached $\mathrm{H}$ atoms, respectively, while the $S$ and $H$ charges are +2.25 and +0.49 , respectively.

\section{ACKNOWLEDGEMENTS}

We thank Professor R. L. Kuczkowski for suggesting this study and for permission to quote the results of the microwave structural determination 
(ref. 1). The assistance of Mr. L. W. Reinish with the calculations and the use of the facilities of the University of Michigan Computing Center are gratefully acknowledged.

\section{REFERENCES}

1 R. L. Kuczkowski, R. D. Suenram and F. J. Lovas, J. Am. Chem. Soc., 103 (1981) 2561.

2 P. M. Holland and A. W. Castelman, Jr., Chem. Phys. Lett., 56 (1978) 511.

3 W. J. Hehre, W. A. Lathan, R. Ditchfield, M. D. Newton and J. A. Pople, Quantum Chemistry Program Exchange, No. 236, 1975.

4 D. F. Bocian, H. M. Pickett, T. C. Rounds and H. L. Strauss, J. Am. Chem. Soc., 97 (1975) 687.

5 L. Radom, W. J. Hehre and J. A. Pople, J. Am. Chem. Soc., 93 (1971) 289.

6 G. A. Jeffrey, J. A. Pople and L. Radom, Carbohydr. Res., 25 (1972) 117.

7 D. G. Gorenstein, B. A. Luxon and J. B. Findlay, Biochim. Biophys. Acta, 475 (1977) 184. 